\title{
Dinâmica das áreas de floresta nativa no Rio Grande do Sul no período de 1988 a 2020
}

\author{
Dynamics for the native forest regions state in Rio Grande do Sul, Brazil, from 1988 to 2020
}

\author{
Elvis Rabuske Hendges ${ }^{I^{*}}$ Rudiney Soares Pereira $^{\mathrm{II}}$ Juliano Andres ${ }^{\mathrm{I}}$
}

\section{RESUMO}

Os padrões de uso da terra e a utilização dos recursos naturais motivam atualmente muitas pesquisas no campo da simulação de cenários em diversas regiões do mundo. Igualmente, o presente trabalho pretende simular o cenário de florestas nativas do Rio Grande do Sul a fim de localizar e quantificar em um mapa a evolução das florestas deste Estado para o ano de 2020. Para tanto, foram classificados os padrões de uso da terra em imagens dos satélites referentes aos anos de 1988, 1998 e 2007. O mapa da evolução florestal, ao ser cruzado com vários mapas temáticos de aspectos físicos $e$ humanos, definiu, respectivamente, a matriz de transição Markov e os pesos de evidências, ambos requisitos na calibração do modelo estocástico. Como resultado, a simulação da paisagem florestal do Rio Grande do Sul para o ano de 2020 apresentou um aumento de áreas de floresta nativa em quase todas as regiões do Estado com mais de $5.113 \mathrm{~km}^{2}$.

Palavras-chave: simulação de cenários, SIG, sensoriamento remoto.

\section{ABSTRACT}

Land use patterns and the potential misuse of our planet's natural resources have recently called the attention of an increasing worldwide number of studies in the area of scenario simulation. In this context, the present study aims at simulating the native forest scenario of the southern state of Rio Grande do Sul, Brazil, for the 2020, by locating and quantifying in a map the evolution of forest in this state. Land use patterns were classified based on satellite images from the years 1988, 1998 and 2007. The forest evolution map was cross-checked with several thematic maps of physical and human aspects and defined the Markov transition matrix and the evidence weights, both of which are requirements for the calibration of the stochastic model. The simulation of Rio Grande do Sul's forest landscape for the year 2020 showed an increase of the native forest in almost all regions of the state, covering an area of more than $5,113 \mathrm{~km}^{2}$.

Key words: scenario simulation, GIS, remote sensing.

\section{INTRODUÇÃO}

A simulação de paisagens vem se apresentando como uma ferramenta muito útil na projeção de cenários futuros e tem incentivado muitas pesquisas nos últimos anos. A preocupação com a degradação de algumas paisagens faz com que a maioria dos modelos de simulação esteja vinculada aos emergentes impactos ambientais, os quais impulsionaram uma série de pesquisas com acompanhamentos dos padrões de uso da terra e dos fatores determinantes nas mudanças de ocupação do solo, denominadas de LUCC (Land Use and Land Cover Change) (STEYAERT, 1993).

Um dos modelos de simulação mais empregados em estudos da paisagem é o modelo estocástico, também chamado de estocástico-

${ }^{\mathrm{I} G}$ Grupo de Pesquisa em Geotecnologias (GEOTEC), Universidade Estadual do Oeste do Paraná (UNIOESTE), 85605-010, Campus de Francisco Beltrão, Francisco Beltrão, PR, Brasil. E-mail: elvis.hendges@unioeste.br. *Autor para correspondência.

IDepartamento de Engenharia Rural, Universidade Federal de Santa Maria (UFSM), Santa Maria, RS, Brasil. 
probabilístico. Através deste, é possível simular as áreas futuras mais susceptíveis a novas alterações de uso, tendo como bases uma matriz de transição definida a partir de mudanças de uso da terra em datas passadas e a vinculação dessas mudanças a certos critérios definidos por pesos de evidências (GOODCHILD, 1992).

No Brasil, estudos de simulação de impacto ambiental e econômico causados pela ocupação da floresta amazônica, realizados por SOARES-FILHO et al. (2002) e AGUIAR et al. (2007), mostram que o desflorestamento está num primeiro momento ligado à abertura de uma estrada principal e à posterior criação de caminhos vicinais, dando à paisagem o padrão de uso conhecido como "espinha de peixe".

São também encontradas pesquisas de simulação estocástica em áreas do cerrado, onde a previsão de queimadas em reservas naturais, provocadas pelo avanço agrícola indiscriminado, ajuda a definir as áreas mais susceptíveis aos incêndios no futuro, buscando, assim, uma melhor manutenção deste ecossistema (MANZIONE et al., 2006).

Ao contrário das paisagens mencionadas anteriormente, a fronteira agrícola do Estado do Rio Grande do Sul já está definida, e o padrão de uso da terra nos últimos anos respeita uma certa padronização quanto à ocupação humana. Assim, a evolução das florestas nativas demonstra particularidade se comparado com a realidade dos desflorestamentos encontrados nos biomas do cerrado e da Amazônia, pois, segundo dados dos inventários florestais dos anos de 1983 e 2001, as áreas de floresta nativa sofreram um acréscimo de mais de 33 mil km², passando de 15.857,31 para 49.556,29 $\mathrm{km}^{2}$ (SEMA, 2010). Dessa forma, o objetivo deste estudo é a criação de um modelo estocástico-probabilístico, com a finalidade de simular o cenário das florestas nativas do Rio Grande do Sul referente ao ano de 2020 .

\section{MATERIAL E MÉTODOS}

Para criação do modelo de simulação, estudaram-se os padrões de uso da terra pelos quais o Rio Grande do Sul foi submetido ao longo dos últimos anos e, para isso, foram classificadas imagens de satélite de três períodos distintos: 1988, 1998 e 2007.

Dessa forma, para a obtenção do uso da terra dos anos de 1988 e 1998, foram classificadas imagens do satélite LANDSAT 5 (sensor TM) e
LANDSAT 7 (sensor ETM+) bandas 3, 4 e 5, de resolução espacial de $30 \mathrm{~m}$, somando um total de 21 cenas por período. As imagens foram importadas individualmente para um Banco de Dados, criado no aplicativo SPRING. Já para o mapa de uso da terra referente a 2007, foram utilizadas imagens do satélite Terra, sensor MODIS, banda 45 e 6 , de resolução espacial de $250 \mathrm{~m}$, sendo necessária a importação de duas imagens para contemplar todo território do Rio Grande do Sul. A mudança quanto ao padrão de imagens utilizadas foi motivada pela ausência da disponibilidade de dados referente aos períodos estudados. A fim de homogeneizar os dados, os mosaicos de imagens de 1988 e 1998 tiveram sua resolução espacial reduzida para compatibilizá-las com as imagens de 2007.

O processo de classificação das imagens levou em conta os padrões de uso da terra, definidas pelo Inventário Florestal Contínuo (Informe Verbal 2). Assim, foi espacializada, sob as áreas de estudo, uma malha amostral de dimensão $3 \times 3$ pixels. Para cada tema, foram analisados os valores de 150 unidades poligonais. A extração dos valores médios para a unidade amostral foi realizada através da função “estatística da imagem por polígonos" do SPRING. Com base nestes valores médios, calculou-se a média aritmética simples entre as leituras de cada imagem selecionada, para que então esses valores fossem tomados como referência para cada período a ser avaliado. Para avaliar a heterogeneidade dos valores médios definidos para os temas a serem classificados numa mesma data e a homogeneidade entre os mesmos temas, mesmo em períodos distintos, aplicou-se o Teste Tukey HSD.

Após definido o intervalo médio dos valores digitais dos pixels referentes a cada tema a ser classificado através do agrupamento mínimo de pixels pertencentes a uma mesma classe de uso da terra, foi realizada a classificação supervisionada com a utilização do algoritmo da máxima verossimilhança (MaxVer), a qualidade de cada uma das classificações foi avaliada através do índice Kappa (LANDIS \& KOCH, 1977).

Já a elaboração dos mapas temáticos teve três bases distintas de informação: (1) 29 cartas topográficas da DSG (Informe Verbal 3), na escala de 1:250.000, que foram georreferenciadas e importadas para o banco de dados no aplicativo SPRING, em que se procedeu à vetorização das redes de drenagem e de estradas de rodagem; (2) levantamento tridimensional 
de elevação da terra elaborado por meio da SRTM (Shuttle Radar Topography Mission) da NASA (National Aeronautics and Space Administration); e (3) tabelas referentes aos censos socioeconômicos realizados pelo Instituto Brasileiro de Geografia e Estatística (IBGE).

Antes de criar o modelo de simulação de cenários, os mapas de mudanças de uso da terra elaborados com imagens de satélite serviram de base para o cálculo das probabilidades de transição da matriz de Markov. De posse desses valores, dos mapas de uso da terra e dos mapas temáticos, procedeu-se à simulação no aplicativo DINAMICA(Informe Verbal 3).

Os mapas temáticos gerados (proximidade das estradas e dos cursos de drenagem; grau de declividade e hipsometria do terreno; contingente populacional; índices de urbanização e desenvolvimento humano; atividades de exploração florestal), são estáticos e tem sua participação no modelo definida pelos cálculos de valores do método de pesos e evidências. As variáveis temáticas são consideradas estáticas durante o processo de simulação, pois a possibilidade de prever alterações desses índices estudados é remota.

\section{RESULTADOS E DISCUSSÃO}

Como primeiro resultado, a presente pesquisa apresentou os dados referentes à heterogeneidade entre as classes de uso da terra selecionadas e a homogeneidade destas nos períodos estudados. Para tal, foi aplicado o teste de Tukey nas médias das classes de uso da terra que envolvem o mapeamento de espécies florestais (principal objeto de estudo). Com limite inferior positivo, o teste mostrou diferenças significativas em nível $\alpha \leq 5 \%$. Para a interpretação dos resultados, deve-se considerar (1) floresta nativa, (2) florestamentos, (3) regeneração e (4) campo. Os resultados indicam que as médias encontradas são distintas em relação ao comportamento espectral de cada classe, sendo, portanto, possível seu uso como indicadoras para a validação da classificação.

Para a classificação digital dos temas, a matriz de confusão e o cálculo do índice Kappa demonstraram-se satisfatórios, uma vez que os valores encontrados foram de $0,98,0,98 \mathrm{e} 0,96$, respectivamente. Para quantificar a precisão da classificação, segundo o índice Kappa, estes se enquadram na faixa de 0,80-1,0, considerada como sendo de excelente qualidade.

O crescimento de áreas do uso referente às florestas nativas novas e regeneradas ao longo dos últimos 20 anos no Estado do Rio Grande do Sul é apresentado na tabela 1. Esse crescimento corresponde a um crescimento médio de $1,15 \%$ por década de análise, em relação à área total desse estado, ou seja, um ganho de aproximadamente $3.237,8 \mathrm{~km}^{2}$ de florestas nativas a cada 10 anos. Isso demonstra que esse estado está em processo de recuperação de áreas florestais, suprindo uma carência de áreas nativas, as quais em 2007 eram de apenas $15 \%$ da área total. A matriz de Markov, parte fundamental do modelo de simulação, foi definida pela média das transições sofridas pelas classes de uso da terra nos períodos estudados (Tabela 2).

Os resultados quantificados do modelo de simulação de aumento ou diminuição das áreas de florestas nativas para o ano de 2020, em relação ao ano

Tabela 1 - Áreas de uso da terra do Rio Grande do Sul em $\mathrm{km}^{2}$.

\begin{tabular}{lcccc}
\hline Classes de Uso & Uso de 1988 & Uso de 1998 & Uso de 2007 & Inv. Florestal \\
\hline Florestas Nativas & $35.850,04$ & $38.982,30$ & $42.245,76$ & $38.159,52$ \\
Florestamento & $3.319,13$ & $4.545,54$ & $5.616,81$ & $2.747,48$ \\
Capoeirão & $10.541,91$ & $12.305,49$ & $14.414,79$ & $11.396,77$ \\
Campo & $133.424,92$ & $133.138,15$ & $127.938,29$ & $132.102,60$ \\
Agriculturas & $76.779,98$ & $69.436,72$ & $67.077,72$ & $71.377,89$ \\
Aflor. Rochoso & 944,49 & $1.023,87$ & $1.076,18$ & - \\
Dunas & $1.510,94$ & $1.461,33$ & $1.502,67$ & $1.655,55$ \\
Água & $16.915,91$ & $18.277,43$ & $20.258,95$ & $20.050,28$ \\
Banhado & $2.261,23$ & $2.377,70$ & $1.417,36$ & $2.018,78$ \\
Total & $281.548,54$ & $281.548,54$ & $281.548,54$ & \\
\hline
\end{tabular}

Ciência Rural, v.42, n.5, mai, 2012. 
Tabela 2 - Matriz de transição de Markov (\%).

\begin{tabular}{|c|c|c|c|c|c|c|c|c|c|}
\hline Uso X Uso & Flo & Reflo & Cap. & Campo & Agric. & Afl. Roch. & Dunas & Água & Banh. \\
\hline Florestas & 75,79 & 1,32 & 2,33 & 6,72 & 13,22 & 0,11 & 0,01 & 0,23 & 0,23 \\
\hline Reflorestamento & 6,28 & 73,76 & 11,38 & 1,81 & 5,86 & 0,14 & 0,19 & 0,18 & 0,35 \\
\hline Capoeirão & 17,47 & 12,41 & 52,65 & 1,30 & 14,59 & 0,14 & 0,12 & 0,74 & 0,53 \\
\hline Campo & 5,64 & 15,40 & 3,42 & 52,37 & 21,84 & 0,68 & 0,43 & 0,17 & 0,37 \\
\hline Agricultura & 1,31 & 17,08 & 34,74 & 9,33 & 35,25 & 0,89 & 0,18 & 0,34 & 0,82 \\
\hline Afl. Rochoso & 2,36 & 15,31 & 2,52 & 5,52 & 13,86 & 57,96 & 1,37 & 1,18 & 0,37 \\
\hline Dunas & 0,99 & 2,33 & 0,67 & 3,26 & 1,48 & 0,58 & 87,84 & 2,39 & 0,46 \\
\hline Água & 0,46 & 2,30 & 0,66 & 4,57 & 0,41 & 0,03 & 0,94 & 82,77 & 7,79 \\
\hline Banhado & 2,87 & 17,84 & 5,90 & 4,47 & 37,87 & 2,29 & 0,69 & 1,71 & 26,33 \\
\hline
\end{tabular}

de 2007, não apresentaram nenhuma mudança muito abrupta, respeitando assim a tendência apresentada pelo padrão de uso florestal encontrados nos períodos de 1988, 1998 e 2007 (Tabela 3).

Em relação ao ganho de área de florestas nativas, a simulação da paisagem referente ao ano de 2020 apontou um aumento de $5.313 \mathrm{~km}^{2}$ em relação ao ano de 2007, sendo maior do que os acréscimos de $3.263 \mathrm{~km}^{2}$ e $3.132 \mathrm{~km}^{2}$ registrados para os períodos de 1998-2007 e 1988-1998, respectivamente. Esse aumento de mais de $5.313 \mathrm{~km}^{2}$ corresponde a, aproximadamente, $2 \%$ de todo território do Rio Grande do Sul. Como constatado anteriormente, a projeção de cenário referente ao uso das classes florestais do Rio Grande do Sul para o ano de 2020 não apresentou, sobre o mapa, uma área de mudança muito perceptível em relação ao volume de alterações, porém, ao analisar a distribuição das novas áreas de florestas nativas, é possível identificar uma maior concentração destas na região noroeste do Rio Grande do Sul (Figura 1).

Tabela 3 - Quantificação das áreas de uso da terra do Rio Grande do Sul em $\mathrm{km}^{2}$, definidas pela simulação estocástica da paisagem.

\begin{tabular}{llc}
\hline Classes de Uso & Uso de 2007 & Simulação 2020 \\
\hline Florestas Nativas & $42.245,76$ & $47.358,85$ \\
Florestamentos & $5.616,81$ & $9.652,49$ \\
Capoeirão & $14.414,79$ & $20.704,21$ \\
Campo & $127.938,29$ & $116.541,65$ \\
Agriculturas & $67.077,72$ & $63.305,27$ \\
Afl. Rochoso & $1.076,18$ & $1.348,55$ \\
Dunas & $1.502,67$ & $1.241,75$ \\
Água & $20.258,95$ & $20.279,28$ \\
Banhado & $1.417,36$ & $1.116,21$ \\
Total & $281.548,54$ & $281.548,54$ \\
\hline
\end{tabular}

Já as áreas de florestas nativas mapeadas em 2007 e que mudaram o uso da terra, passando a pertencer a outra classe, totalizaram uma perda de $6.977 \mathrm{~km}^{2}$, respeitando assim uma tendência menor relacionada ao desmatamento de áreas com cobertura de florestas nativas, pois este tipo de mudança de uso da terra entre os anos de 1988-1998 representava uma área de $9.601 \mathrm{~km}^{2}$ e, no período seguinte, 1998-2007, apresentou uma diminuição, passando a ser de $8.435 \mathrm{~km}^{2}$. Dessa forma, a classe de uso da terra que mais influenciou no desmatamento de florestas nativas no mapa de simulação para o ano de 2020 foi a agricultura, com cerca de $2.987 \mathrm{~km}^{2}$, representando aproximadamente $42 \%$ do total das áreas desmatadas. A tabela 4 traz o total das áreas que em 2007 eram classificadas como floresta nativa e que, de acordo com o mapa resultante do modelo de simulação estocástico da evolução florestal do Rio Grande do Sul, referente ao ano de 2020, passaram a pertencer as outras classes de uso da terra. Os valores mencionados, quando espacializados sobre o território do Rio Grande do Sul, apresentam uma concentração de áreas na região central do Estado, onde o desmatamento apresentou maior intensidade no mapa de simulação (Figura 2).

\section{CONCLUSÃO}

Com os procedimentos metodológicos adotados, pode-se concluir que o modelo de simulação da evolução das áreas florestais nativas no Rio Grande do Sul mostrou uma continuidade em relação ao padrão de uso da terra que vem sendo adotado nos últimos anos neste Estado, sendo que o aumento das áreas de floresta nativa apresenta um crescimento proporcional 


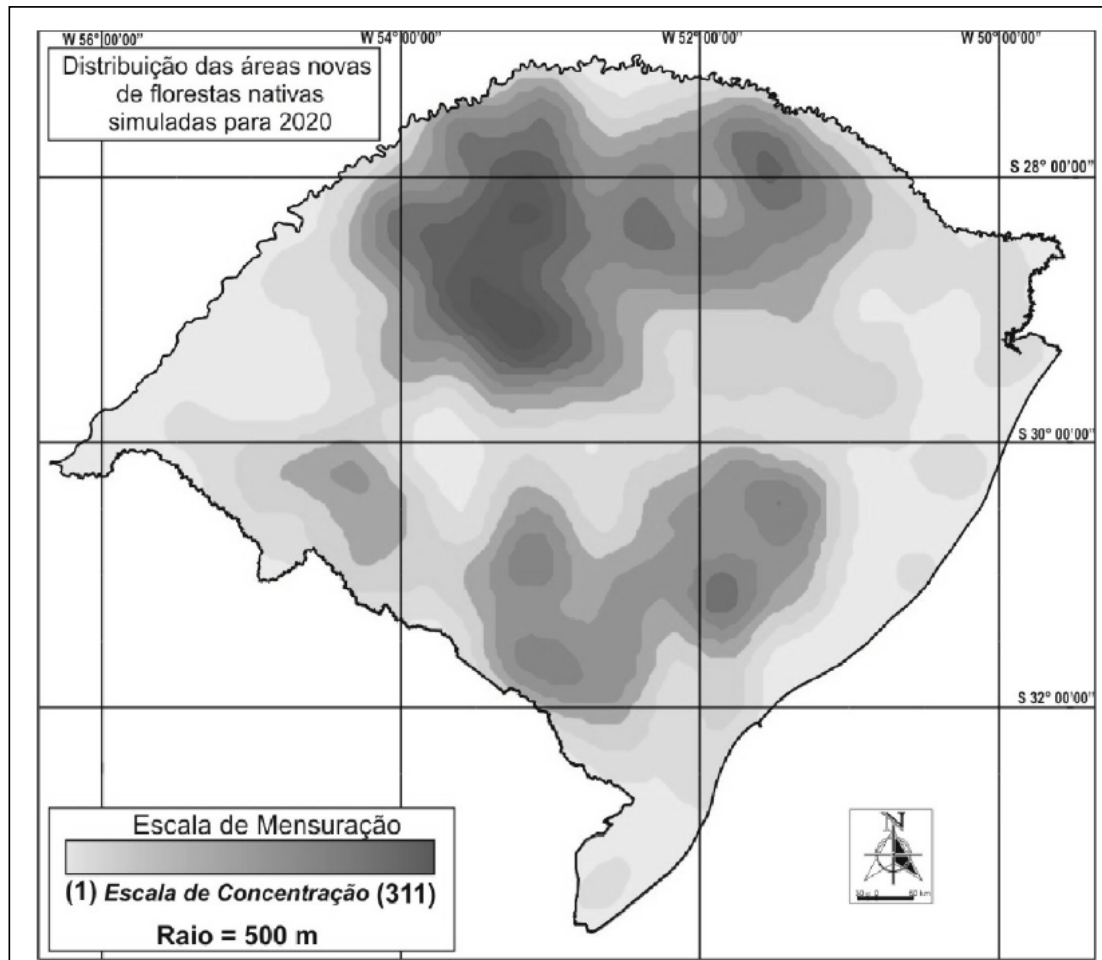

Figura 1 - Distribuição das novas áreas de floresta nativa simuladas para 2020.

com o passar dos anos. Já a atividade de desmatamento, na simulação referente ao ano de 2020, apresentou uma redução, comparada ao período anterior estudado.

\section{AGRADECIMENTOS}

À Coordenação de Aperfeiçoamento de Pessoal de Nível Superior (CAPES), pelo apoio financeiro para o desenvolvimento da pesquisa.

Ao Programa de Pós-graduação de Engenharia Florestal (PPGEF) da Universidade Federal de Santa Maria (UFSM), por oportunizar a realização da pesquisa.

\section{INFORME VERBAL}

O Inventário Florestal Contínuo do Rio Grande do Sul foi realizado entre os anos 1998 e 2001 com o objetivo de avaliar quantitativa e qualitativamente os recursos florestais do Rio Grande do Sul.

A Diretoria de Serviço Geográfico (DSG) é o órgão de apoio setorial, na estrutura do Exército destinado à elaboração de produtos cartográficos.

O DINAMICA é um aplicativo que gera modelos de simulação de paisagens de domínio público, desenvolvido pelo Centro de Sensoriamento Remoto da Universidade Federal de Minas Gerais.

Tabela 4 - Classes de uso da terra que contribuíram na diminuição das áreas de florestas nativas mapeadas em 2007 (simulação do ano de 2020).

\begin{tabular}{lcc}
\hline Classes de Uso & Transformação em km & Porcentagem (\%) do total \\
\hline Reflorestamento & 629,86 & 9,027 \\
Capoeirão & 782,37 & 11,213 \\
Campo & $2.572,39$ & 36,868 \\
Agriculturas & $2.987,22$ & 42,813 \\
Afl. Rochoso & 0,43 & 0,006 \\
Dunas & 0,06 & 0,001 \\
Água & 0,24 & 0,003 \\
Banhado & 4,88 & 0,070 \\
Total & $6.977,44$ & 100 \\
\hline
\end{tabular}




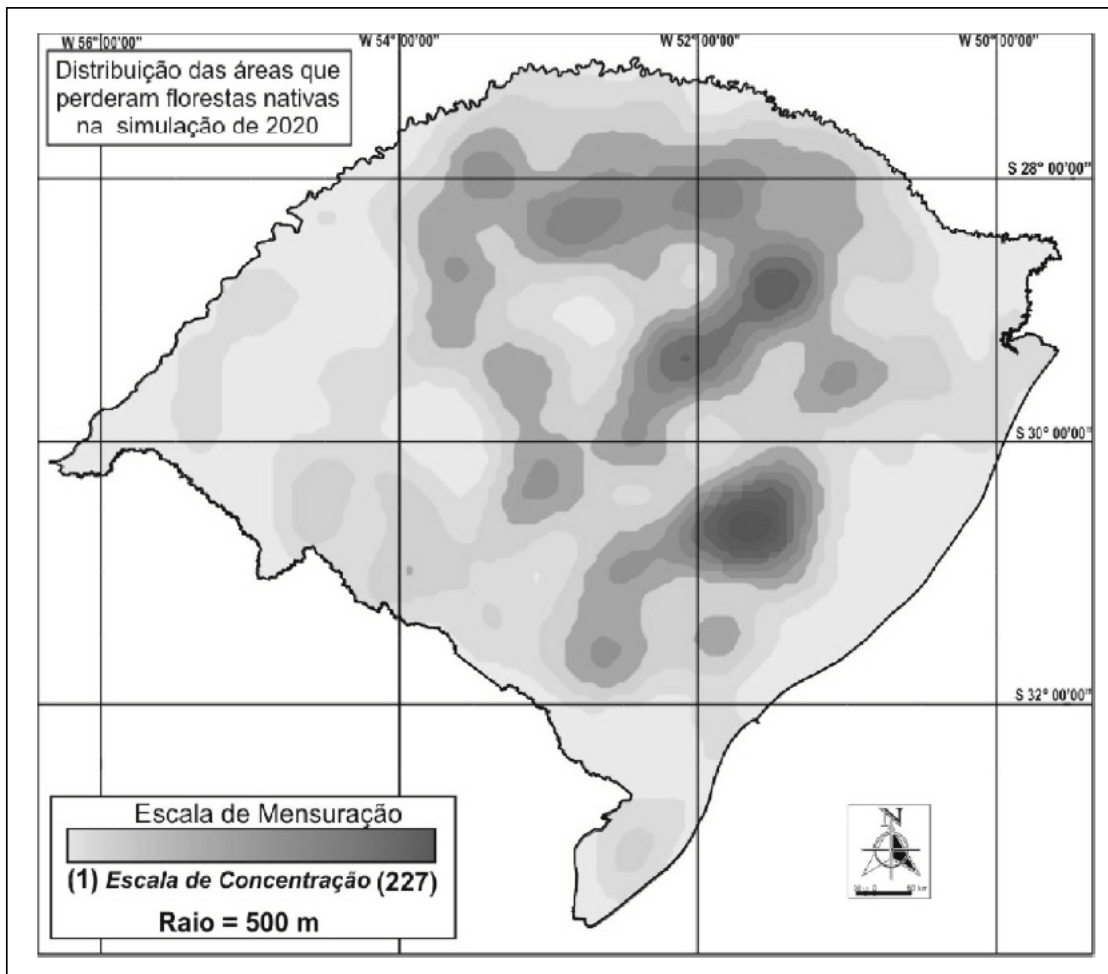

Figura 2 - Distribuição das áreas de desflorestamento (2020).

\section{REFERÊNCIAS}

AGUIAR, A.P.D. et al. Spatial statistical analysis of land-use determinants in the Brazilian Amazonia: Exploring regional heterogeneity. Ecological Modelling, v.209, p.169-188, 2007. Disponível em: <http://www.dpi.inpe.br/gilberto/papers/ amazon_ecomod.pdf>. Acesso em: 03 jun. 2008. doi: 10.1016/j.

GOODCHILD, M. F. Geographical data modeling. Computers \& Geosciences, v.4, n.18, p.401-408, 1992. Disponível em: $<$ http://www.geog.ucsb.edu/ good/papers/172.pdf $>$. Acesso em: 25 nov. 2006. doi: 10.1016/0098-3004(92)90069-4.

LANDIS, J. R.; KOCH, G. G. The measurement of observer agreement for categorical data. Biometrics, v.33, n.1, p.159$174,1977$.

MCGARIGAL, K.; MARKS, B.J. Fragstat: spatial pattern analysis program for quantifying landscape struture. Portland, US: Department of Agriculture, Forest Service, Pacific Northwest Research Station, 1995. 132p.
MANZIONE, R. L. et al. Predictive risk mapping of water table depths in a Brazilian Cerrado Area. In: STEIN, A. et al. Quality aspects in spatial data mining. Florida: CRC, 2008. Cap.7, p.73-89.

SEMA. Cobertura florestal. Disponível em: <http:// www.sema.rs.gov.br/sema/html/cobflinve2.htm $>$. Online. Acesso em: 26 ago. 2010

SOARES-FILHO, B.S. et al. Dinamica - a stochastic cellular automata model designed to simulate the landscape dynamics in an Amazonian colonization frontier. Ecological Modelling, v.154 n.3, p.217-235, 2002. Disponível em: <http:// www.dpi.inpe.br/gilberto/cursos/modelling/dinamica.pdf $>$. Acesso em: 05 set. 2006. doi: 10.1016/S0304-3800(02)00059-5.

STEYAERT, L. A. Perspective on the state of environmental simulation modeling. New York: Oxford University, 1993. $167 \mathrm{p}$. 